$10-1-2000$

\title{
Exterior Caustics Produced in Scattering of a Diagonally Incident Plane Wave by a Circular Cylinder: Semiclassical Scattering Theory Analysis
}

James A. Lock

Cleveland State University, j.lock@csuohio.edu

Epltarkeshis. Axdeddditional works at: https://engagedscholarship.csuohio.edu/sciphysics_facpub

Eaward $A$. the Physics Commons

How does access to this work benefit you? Let us know!

\section{Publisher's Statement}

This paper was published in Journal of the Optical Society of America A: Optics Image Science and Vision and is made available as an electronic reprint with the permission of OSA. The paper can be found at the following URL on the OSA website: http://www.opticsinfobase.org/josaa/ abstract.cfm?URI=josaa-17-10-1846. Systematic or multiple reproduction or distribution to multiple locations via electronic or other means is prohibited and is subject to penalties under law.

\section{Original Citation}

Lock, James A., Charles L. Adler, and Edward A. Hovenac. "Exterior Caustics Produced in Scattering of a Diagonally Incident Plane Wave by a Circular Cylinder: Semiclassical Scattering Theory Analysis." Journal of the Optical Society of America A: Optics Image Science and Vision 17 (2000): 1846-1856.

\section{Repository Citation}

Lock, James A.; Adler, Charles L.; and Hovenac, Edward A., "Exterior Caustics Produced in Scattering of a Diagonally Incident Plane Wave by a Circular Cylinder: Semiclassical Scattering Theory Analysis" (2000). Physics Faculty Publications. 103.

https://engagedscholarship.csuohio.edu/sciphysics_facpub/103

This Article is brought to you for free and open access by the Physics Department at EngagedScholarship@CSU. It has been accepted for inclusion in Physics Faculty Publications by an authorized administrator of EngagedScholarship@CSU. For more information, please contact library.es@csuohio.edu. 


\title{
Exterior caustics produced in scattering of a diagonally incident plane wave by a circular cylinder: semiclassical scattering theory analysis
}

\author{
James A. Lock \\ Department of Physics, Cleveland State University, Cleveland, Ohio 44115 \\ Charles L. Adler \\ Department of Physics, St. Mary’s College of Maryland, St. Mary’s City, Maryland 20686 \\ Edward A. Hovenac \\ Federal Data Corp., NASA Glenn Research Center, Cleveland, Ohio 44135
}

Received October 22, 1999; revised manuscript received May 15, 2000; accepted May 30, 2000

\begin{abstract}
We use the semiclassical limit of electromagnetic wave scattering theory to determine the properties of the exterior caustics of a diagonally incident plane wave scattered by an infinitely long homogeneous dielectric circular cylinder in both the near zone and the far zone. The transmission caustic has an exterior/interior cusp transition as the tilt angle of the incident beam is increased, and each of the rainbow caustics has a farzone rainbow/exterior cusp transition and an exterior/interior cusp transition as the incident beam tilt angle is increased. We experimentally observe and analyze both transitions of the first-order rainbow. We also compare the predictions of the semiclassical approximation with those of ray theory and exact electromagnetic wave scattering theory. (c) 2000 Optical Society of America [S0740-3232(00)01010-3]
\end{abstract}

OCIS codes: $080.1510,290.4020$.

\section{INTRODUCTION}

When an electromagnetic plane wave of wavelength $\lambda$ is incident on an infinitely long homogeneous dielectric circular cylinder of radius $a$ in the short-wavelength limit $\lambda \ll a$, the scattered light contains a number of optical caustics. For normal incidence, light transmitted through the cylinder forms a cusp-shaped focusing caustic in the near zone, called the $p=1$ caustic. Light transmitted through the cylinder following $p-1 \geqslant 1$ internal reflections forms the two curving branches of the $(p-1)$-order rainbow caustic that extend from the cylinder surface out to infinity. The $p=1$ focusing caustic and the $p \geqslant 2$ rainbow caustics appear to be quite different structures. But in the context of catastrophe theory, ${ }^{1}$ they are merely different manifestations of the same general caustic morphology if the assumptions of normal incidence and plane waves are relaxed. For example, if a plane wave illuminates the cylinder at a progressively steeper angle of incidence or, equivalently, if the refractive index of the cylinder progressively increases, ${ }^{2}$ the two branches of the far-zone $(p-1)$-order rainbow caustic approach each other, join together at infinity ${ }^{3-7}$ in what has been called the caustic merging transition, and evolve into a near-zone focusing caustic. If the angle of incidence is further increased, the focal line of the near-zone caustic approaches the cylinder and touches its surface, and the caustic evolves into a cusp lying entirely within the cylinder. This is called the exterior/interior cusp transition.

The same progression occurs for the $p=1$ focusing caustic. As the tilt angle of the incident plane wave progressively increases from normal incidence, the focal line of the $p=1$ caustic approaches the cylinder and touches its surface, and the caustic evolves into an interior cusp. ${ }^{3}$ If the cylinder is illuminated by the diverging beam of a line source that is brought progressively closer to the cylinder, the focal line of the $p=1$ caustic moves out to infinity, and the caustic opens up into the two branches of the $p=1$ rainbow. ${ }^{8}$

In this paper we use a simple version of the semiclassical limit of electromagnetic wave scattering theory, ${ }^{9,10}$ to examine the $p \geqslant 1$ caustics of a circular cylinder illuminated by a diagonally incident plane wave. The calculations presented here have a number of basic motivations. First, the complete morphology of cylinder caustics is easily observed experimentally with a minimum of laboratory equipment. All one has to do is place the cylinder in the incident beam, progressively tilt the cylinder, and watch the caustics evolve.

These caustics are also important in the fluid diagnostic technique known as rainbow refractometry, which allows one to obtain the refractive index and the diameter 
of a liquid droplet ${ }^{11,12}$ or column ${ }^{13}$ from the first-order rainbow angle and the periodicity of its supernumerary interference pattern. When this technique is employed, the detector array is often out of necessity placed in the scattering near zone, where the rainbow angle and supernumerary periodicity differ substantially from their farzone values. If measurements made in the near zone are not corrected, the inferred refractive index and diameter will be in error. The shift in the rainbow angle in the near zone has been calculated by using ray theory. ${ }^{8}$ In this paper we derive the near-zone shift in the supernumerary periodicity and a simpler expression for the nearzone shift of the rainbow angle.

Our third motivation is the usefulness of the semiclassical approximation for calculating all the caustic properties in a relatively straightforward way. For example, one may determine the shape of the $p \geqslant 1$ scattering caustics of a cylinder by using ray theory, since a caustic is the envelope of the $p$-family of scattered rays. ${ }^{14}$ The supernumerary interference structure associated with rainbow caustics in the $r \rightarrow \infty$ far-zone limit is usually calculated by using Airy theory, ${ }^{15}$ and the diffraction structure associated with the $p=1$ near-zone focusing caustic may be calculated by using Fourier optics methods. ${ }^{16}$ The semiclassical approximation, on the other hand, allows one to determine all the properties of all the caustics in a unified way rather than by using a patchwork of different methods for different caustic properties at different distances from the cylinder.

The body of this paper is organized as follows. In Subsection 2.A we describe the plane-wave-cylinder geometry, and in Subsection 2.B we summarize the near-zone solution to the electromagnetic boundary-value problem of a diagonally incident plane wave scattered by an infinitely long homogeneous dielectric circular cylinder. In Subsection 2.C we obtain the semiclassical limit of the $p \geqslant 1$ terms of the Debye-series expansion of the nearzone scattered fields. We then use these results in Sections 3 and 4 to examine the $p=1$ and $p \geqslant 2$ caustics, respectively. Wherever possible, we compare our results with those obtained by other methods. In Subsections 4.B and 4.C, we describe our experimental observation of the $p=2$ caustic merging transition and exterior/interior cusp transition. Finally, in Section 5 we summarize our results.

\section{NEAR-ZONE SCATTERING OF A DIAGONALLY INCIDENT PLANE WAVE BY A DIELECTRIC CIRCULAR CYLINDER}

The formulas describing scattering of a diagonally incident plane wave or focused beam by a homogeneous dielectric circular cylinder are given in Ref. 17, and the Debye-series decomposition of the diagonal-incidence partial-wave scattering amplitudes is given in Ref. 4 . In this section we extend these far-zone results to the nearzone scattered fields. Our notation follows that of Refs. 4 and 17.

\section{A. Beam-Cylinder Geometry}

The geometry of the diagonally incident plane wave and the dielectric circular cylinder is illustrated in Fig. 1.
The cylinder has radius $a$ and real refractive index $n$, and the cylinder axis coincides with the $z$ axis of a rectangular coordinate system. The incident plane wave has time dependence $\exp (-i \omega t)$, wavelength $\lambda$, wave number $k=2 \pi / \lambda$ and electric field strength $E_{0}$, and its propagation direction is in the $x z$ plane at an angle $\xi$ below the positive $x$ axis. The scattering angle in the $x y$ plane with respect to the positive $x$ axis is $\theta$, and the scattering angle above the $x y$ plane is $\eta$. For diagonal plane-wave incidence the direction of the scattered wave is $\eta=-\xi$. The incident and scattered fields are $\epsilon$-polarized if the electric field vector lies in the $x y$ plane, and they are $\mu$-polarized if the magnetic field vector lies in the $x y$ plane. The scattered fields are expressed in terms of the cylindrical coordinates $(r, \theta, z)$.

\section{B. Near-Zone Scattered Fields}

The partial-wave scattering amplitudes for a diagonally incident $\epsilon$-polarized plane wave producing $\epsilon$-polarized scattered waves are $a_{l}(\sin \xi)$, where $l$ is the integer partial wave number with $-\infty<l<\infty$. Similarly, the partial-wave scattering amplitudes for an incident $\mu$-polarized plane wave producing $\mu$-polarized scattered waves are $b_{l}(\sin \xi)$, and the amplitudes for an $\epsilon$-polarized plane wave producing $\mu$-polarized scattered waves or a $\mu$-polarized plane wave producing $\epsilon$-polarized scattered waves are $q_{l}(\sin \xi)$. Detailed formulas for $a_{l}(\sin \xi)$, $b_{l}(\sin \xi)$, and $q_{l}(\sin \xi)$ are given in Eqs. (14) and (15) of Ref. 17 and contain Bessel, Neumann, and Hankel functions evaluated at

$$
\begin{aligned}
& x=k a \cos \xi, \\
& y=n^{\prime} x,
\end{aligned}
$$

where

$$
n^{\prime}=\left(n^{2}-\sin ^{2} \xi\right)^{1 / 2} / \cos \xi
$$

(The size parameters $x$ and $y$ are not to be confused with the $x$ and $y$ axes.) The quantity $n^{\prime}$ is known as the Bravais refractive index of the cylinder. ${ }^{5-7}$

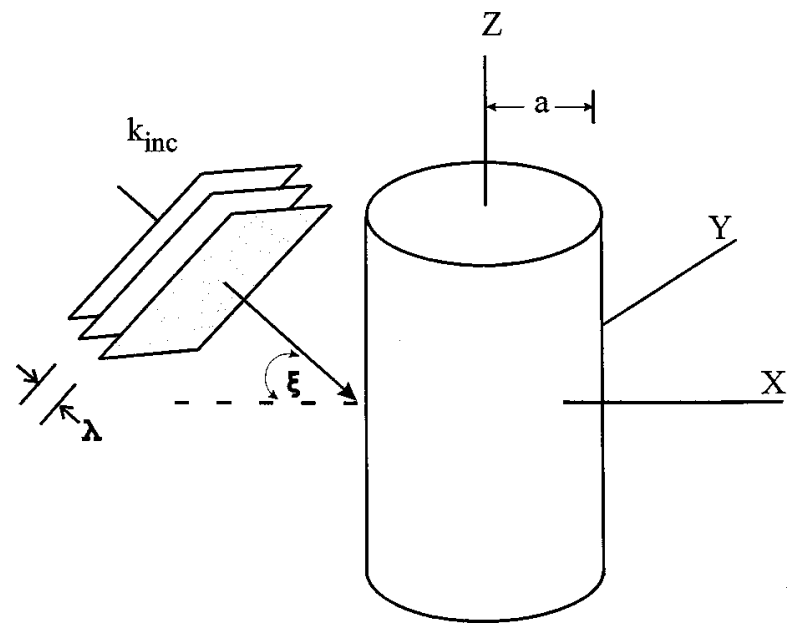

Fig. 1. Plane wave of wavelength $\lambda$ incident with the angle $\xi$ on an infinitely long circular cylinder of radius $a$. 
The near-zone scattered fields for diagonal plane-wave incidence are obtained by combining Eqs. (2), (5), (8), (22), and (24) of Ref. 17. For an $\epsilon$-polarized incident plane wave, we have

$$
\begin{aligned}
& \mathbf{E}_{\text {scatt }}(r, \theta, z) \\
& =i E_{0} \exp (-i k z \sin \xi) \\
& \quad \times \sum_{l=-\infty}^{\infty}\left\{\hat{u}_{r}\left[-(l / k r) \sec \xi P_{l}(r, \theta) a_{l}-\sin \xi Q_{l}(r, \theta) q_{l}\right]\right. \\
& \quad+\hat{u}_{\theta}\left[Q_{l}(r, \theta) a_{l}-(l / k r) \tan \xi P_{l}(r, \theta) q_{l}\right] \\
& \left.\quad+\hat{u}_{z}\left[-\cos \xi P_{l}(r, \theta) q_{l}\right]\right\}, \\
& c \mathbf{B}_{\mathrm{scatt}}(r, \theta, z) \\
& =i E_{0} \exp (-i k z \sin \xi) \\
& \quad \times \sum_{l=-\infty}^{\infty}\left\{\hat{u}_{r}\left[\sin \xi Q_{l}(r, \theta) a_{l}-(l / k r) \sec \xi P_{l}(r, \theta) q_{l}\right]\right. \\
& \quad+\hat{u}_{\theta}\left[(l / k r) \tan \xi P_{l}(r, \theta) a_{l}+Q_{l}(r, \theta) q_{l}\right] \\
& \left.\quad+\hat{u}_{z}\left[\cos \xi P_{l}(r, \theta) a_{l}\right]\right\},
\end{aligned}
$$

where

$$
\begin{aligned}
& P_{l}(r, \theta)=i^{l+1} H_{l}^{(1)}(k r \cos \xi) \exp (i l \theta), \\
& Q_{l}(r, \theta) \equiv i^{l} H_{l}^{(1) '}(k r \cos \xi) \exp (i l \theta) .
\end{aligned}
$$

For a $\mu$-polarized incident plane wave, we have

$$
\begin{aligned}
& \mathbf{E}_{\text {scatt }}(r, \theta, z) \\
&=i E_{0} \exp (-i k z \sin \xi) \\
& \quad \quad \sum_{l=-\infty}^{\infty}\left\{\hat{u}_{r}\left[\sin \xi Q_{l}(r, \theta) b_{l}-(l / k r) \sec \xi P_{l}(r, \theta) q_{l}\right]\right. \\
&+\hat{u}_{\theta}\left[(l / k r) \tan \xi P_{l}(r, \theta) b_{l}+Q_{l}(r, \theta) q_{l}\right] \\
&\left.+\hat{u}_{z}\left[\cos \xi P_{l}(r, \theta) b_{l}\right]\right\}, \\
& c \mathbf{B}_{\text {scatt }}(r, \theta, z) \\
&=i E_{0} \exp (-i k z \sin \xi) \\
& \quad \times \sum_{l=-\infty}^{\infty}\left\{\hat{u}_{r}\left[(l / k r) \sec \xi P_{l}(r, \theta) b_{l}+\sin \xi Q_{l}(r, \theta) q_{l}\right]\right. \\
& \quad+\hat{u}_{\theta}\left[-Q_{l}(r, \theta) b_{l}+(l / k r) \tan \xi P_{l}(r, \theta) q_{l}\right] \\
&\left.\quad+\hat{u}_{z}\left[\cos \xi P_{l}(r, \theta) q_{l}\right]\right\} .
\end{aligned}
$$

The $r \rightarrow \infty$ far-zone limit of the scattered fields is obtained by deleting all terms proportional to $l / k r$ and using the far-zone asymptotic expansion of the Hankel function, ${ }^{18}$

$$
H_{l}^{(1)}(u) \approx(2 / \pi u)^{1 / 2} \exp [i(u-l \pi / 2-\pi / 4)],
$$

which simplifies $P_{l}(r, \theta)$ and $Q_{l}(r, \theta)$ to

$$
\begin{aligned}
P_{l}(r, \theta) \approx & Q_{l}(r, \theta) \\
\approx & (2 / \pi k r \cos \xi)^{1 / 2} \\
& \quad \times \exp [i(k r \cos \xi+l \theta-\pi / 4)] .
\end{aligned}
$$

The normal-incidence limit of the near-zone fields is obtained by setting $\xi=0$ and $q_{l}=0$ in Eqs. (4) and (6).

\section{Semiclassical Limit of the $p \geqslant 1$ Debye-Series Terms for Near-Zone Scattering}

In this subsection we implement the semiclassical approximation following the procedure of Ford and Wheeler. ${ }^{9}$ First, we perform a Debye-series decomposition of the partial-wave scattering amplitudes, ${ }^{4,19}$ which separates the contributions of diffraction plus reflection $(p=0)$, transmission $(p=1)$, and transmission following $p-1$ internal reflections $(p \geqslant 2)$. Next, we approximate the Hankel functions appearing in each Debyeseries term by their asymptotic forms. We then convert the sum over partial waves into a phase integral over an associated impact parameter, since a large number of partial waves contribute to the scattered fields in the smallwavelength limit. Finally, a Taylor-series expansion of the phase of the integrand about a suitable reference point produces either the Airy integral ${ }^{20}$ describing the fold caustic ${ }^{1}$ and its associated diffraction structure (i.e., rainbows) or the Pearcey integral ${ }^{21}$ describing the cusp caustic $^{1}$ and its associated diffraction structure (i.e., nearzone focusing caustics). The Airy and Pearcey integrals are described in Appendix A. The complex angular momentum formalism ${ }^{22-26}$ handles the semiclassical limit of wave scattering in a much more careful and precise way. Our procedure, though not as rigorous as the complex angular momentum formalism, is sufficient to quantitatively describe the major features of the scattering caustics in both the near zone and the far zone.

To make contact with the phase integral representation of caustics, we must pay special attention to the $l$-dependence of the phase of the near-zone scattered fields. In Eqs. (4) and (6), each component of the scattered electric and magnetic field depends on $l$ through $P_{l}(r, \theta)$ and $Q_{l}(r, \theta)$ of Eqs. (5) and the partial-wave scattering amplitudes $a_{l}(\sin \xi), b_{l}(\sin \xi)$, and $q_{l}(\sin \xi)$. We consider first the $l$-dependence of $P_{l}(r, \theta)$ and $Q_{l}(r, \theta)$. In the near zone, the appropriate asymptotic expansion of the Hankel functions in Eqs. (5) is ${ }^{27}$

$$
\begin{aligned}
H_{l}^{(1,2)}(u) \approx & (2 / \pi u)^{1 / 2}\left(1-l^{2} / u^{2}\right)^{-1 / 4} \\
& \times \exp \left\{ \pm i\left[u\left(1-l^{2} / u^{2}\right)^{1 / 2}\right.\right. \\
& +l \arcsin (l / u)-l \pi / 2-\pi / 4]\}, \\
H_{l}^{(1,2) \prime}(u) \approx & \pm i(2 / \pi u)^{1 / 2}\left(1-l^{2} / u^{2}\right)^{1 / 4} \\
& \times \exp \left\{ \pm i\left[u\left(1-l^{2} / u^{2}\right)^{1 / 2}\right.\right. \\
& +l \arcsin (l / u)-l \pi / 2-\pi / 4]\},
\end{aligned}
$$

where the upper and lower signs correspond to $H_{l}^{(1)}$ and $H_{l}^{(2)}$, respectively. Relation (7) is the $(l / u) \rightarrow 0$ limit of relation (9a). When relations (9) are inserted into Eqs. (5), we obtain

$$
\begin{aligned}
P_{l}(r, \theta) \approx & Q_{l}(r, \theta) \approx(2 / \pi k r \cos \xi)^{1 / 2} \\
& \times \exp \left(i x \left\{(r / a)\left[1-w^{2} /(r / a)^{2}\right]^{1 / 2}\right.\right. \\
& +w \arcsin [w /(r / a)]+w \theta\}+i \pi / 4),
\end{aligned}
$$


where the size parameter $x$ was defined in Eq. (1) and $w$ is the impact parameter of the incident ray associated with the partial wave $l$ by using van de Hulst's localization principle ${ }^{28}$

$$
w=l / x .
$$

We next consider the $p$-term of the Debye-series decomposition of the partial-wave scattering amplitudes, denoted here by []$_{p}$. Detailed formulas for the various Debye-series terms are given in Eqs. (22)-(25), (31), and (32) of Ref. 4. The $p$-term of the series is of the form

$$
\left.\begin{array}{l}
{\left[a_{l}(\sin \xi)\right]_{p}} \\
{\left[b_{l}(\sin \xi)\right]_{p}} \\
{\left[q_{l}(\sin \xi)\right]_{p}}
\end{array}\right\}=-\left(\frac{1}{2}\right) \sum T_{l}^{21}\left(R_{l}^{11}\right)^{p-1} T_{l}^{12},
$$

where the transmission and reflection Fresnel coefficients of the partial wave $l$ in each of the four polarization channels $\epsilon \rightarrow \epsilon, \mu \rightarrow \mu, \epsilon \rightarrow \mu$, and $\mu \rightarrow \epsilon$ are $T_{l}^{21}, R_{l}^{11}$, and $T_{l}^{12}$, where the polarization channel indices have been suppressed. These coefficients give the magnitude and the phase of transmission of a radially incoming partial wave from the region outside the cylinder (region 2 ) to the cylinder interior (region 1), of internal reflection of a radially outgoing partial wave in region 1 by the cylinder surface, and of transmission of a radially outgoing partial wave from the cylinder interior to the outside region. The sum in Eq. (12) is over all intermediate polarization states, as described in Ref. 4.

Substituting relations (9) into the expressions for $T_{l}^{21}$, $R_{l}^{11}$, and $T_{l}^{12}$ of Ref. 4 , we find that the phase is independent of polarization channel, i.e.,

$$
\begin{aligned}
& T_{l, \epsilon \epsilon}^{21} \propto T_{l, \mu \mu}^{21} \propto T_{l, \epsilon \mu}^{21} \propto T_{l, \mu \epsilon}^{21} \propto \exp \left(i \phi_{l}^{21}\right), \\
& R_{l, \epsilon \epsilon}^{11} \propto R_{l, \mu \mu}^{11} \propto R_{l, \epsilon \mu}^{11} \propto R_{l, \mu \epsilon}^{11} \propto \exp \left(i \phi_{l}^{11}\right), \\
& T_{l, \epsilon \epsilon}^{12} \propto T_{l, \mu \mu}^{12} \propto T_{l, \epsilon \mu}^{12} \propto T_{l, \mu \epsilon}^{12} \propto \exp \left(i \phi_{l}^{21}\right),
\end{aligned}
$$

where

$$
\begin{aligned}
\phi_{l}^{21}= & y\left(1-l^{2} / y^{2}\right)^{1 / 2}+l \arcsin (l / y) \\
& -x\left(1-l^{2} / x^{2}\right)^{1 / 2}-l \arcsin (l / x), \\
\phi_{l}^{11}= & 2 y\left(1-l^{2} / y^{2}\right)^{1 / 2}+2 l \arcsin (l / y)-l \pi .
\end{aligned}
$$

The magnitude of $T_{l}^{21}, R_{l}^{11}$, and $T_{l}^{12}$ is a slowly varying function of $l$, assumed to be not near zero. For the remainder of this section, we denote all slowly varying magnitudes by $A_{l}$ when considering sums over partial waves and by $A(w)$ when considering integrals over the associated impact parameter.

With the use of Eq. (12), relation (13), and Eqs. (14), the phase of the $p$-term of the Debye series is

$\left[a_{l}\right]_{p} \propto\left[b_{l}\right]_{p} \propto\left[q_{l}\right]_{p} \propto \exp \left\{i\left[2 \phi_{l}^{21}+(p-1) \phi_{l}^{11}\right]\right\}$.

Inserting relations (10) and (15) into Eqs. (4) and (6) and following the procedure of Ref. 9, we obtain

$$
\begin{aligned}
E_{\text {scatt }} & \propto c B_{\text {scatt }} \propto \sum_{l=-\infty}^{\infty} A_{l} \exp \left(i \Phi_{l}\right) \\
& \propto \int_{-\infty}^{\infty} \mathrm{d} w A(w) \exp [i \Phi(w)],
\end{aligned}
$$

where

$$
\begin{aligned}
\Phi(w)= & x\left\{2 p n^{\prime}\left(1-w^{2} / n^{\prime 2}\right)^{1 / 2}+2 p w \arcsin \left(w / n^{\prime}\right)\right. \\
& -2\left(1-w^{2}\right)^{1 / 2}-2 w \arcsin w+(r / a) \\
& \times\left[1-w^{2} /(r / a)^{2}\right]^{1 / 2}+w \arcsin [w /(r / a)] \\
& +w[\theta-(p-1) \pi]\} .
\end{aligned}
$$

In a small interval about a suitably chosen reference point $w_{0}$, the slowly varying magnitude factor $A(w)$ can be brought outside the integral, giving

$$
E_{\text {scatt }} \propto c B_{\text {scatt }} \propto A\left(w_{0}\right) \int_{-\infty}^{\infty} \mathrm{d} w \exp [i \Phi(w)] .
$$

The stationary phase condition

$$
\mathrm{d} \Phi(w) / \mathrm{d} w=0,
$$

applied to Eq. (17), gives

$$
\begin{aligned}
\theta= & (p-1) \pi+2 \arcsin w \\
& -2 p \arcsin \left(w / n^{\prime}\right)-\arcsin [w /(r / a)] .
\end{aligned}
$$

The solution of Eq. (20) for $w$ as a function of $r$ and $\theta$ gives the impact parameter of the incident ray, which, after interacting with the cylinder, passes through the point $(r, \theta)$ on its way to the far zone. This is the generalization of van de Hulst's localization principle to the near zone.

\section{SHAPE OF THE $p=1$ CAUSTIC}

In ray theory the portion of the $p=1$ near-zone transmission caustic in the vicinity of the cusp focal line is produced by the constructive interference of paraxial rays. Thus in the semiclassical approximation we obtain the caustic and its surrounding diffraction structure by assuming that the important partial waves $l$ or impact parameters $w$ are small, i.e., $l \ll x$ or $w \ll 1$. We expand the phase function of Eq. (17) for $p=1$ in powers of $w$ and retain terms of order $w^{4}$ or less so as to include the three stationary points of $\Phi(w)$ (i.e., the three rays) that contribute to the near-zone caustic. We call this truncation of $\Phi(w)$ at order $w^{4}$ the Pearcey approximation of the phase function. With a suitable change of variable, each component of the scattered electric and magnetic field, expressed in terms of $r$ and $\theta$, is proportional to the Pearcey integral of Eq. (A2) with

$$
\begin{aligned}
& X=(24 k r \cos \xi)^{1 / 2}\left[\left(n^{\prime}-1\right) / n^{\prime}\right][(f-r) / a] / D^{1 / 2}, \\
& Y=(24)^{1 / 4}(k r \cos \xi)^{3 / 4} \theta / D^{1 / 4},
\end{aligned}
$$

where

$$
D=\left[2(r / a)^{3}\left(n^{\prime 3}-1\right) / n^{\prime 3}\right]-1 .
$$

The position of the focal line of the cusp caustic is

$$
f / a=n^{\prime} /\left[2\left(n^{\prime}-1\right)\right] .
$$

The exterior/interior cusp transition occurs at $n^{\prime}=2$. The shape of the focusing caustic in the Pearcey approximation is given in cylindrical coordinates by Eq. (A3), or

$$
\theta_{\text {caustic }}= \pm(8 / 3)\left[\left(n^{\prime}-1\right) / n^{\prime}\right]^{3 / 2}\left[\left(f-r_{\text {caustic }}\right) / a\right]^{3 / 2} / D^{1 / 2} \text {. }
$$


Writing

$$
r_{\text {caustic }} / a=f / a-\delta
$$

near the focal line and expanding Eq. (24) in powers of $\delta$, we obtain

$$
\begin{aligned}
\theta_{\text {caustic }} \approx & \pm 16\left(n^{\prime}-1\right)^{5 / 2}\left(3 n^{\prime}\right)^{-3 / 2} \\
& \times\left(-n^{\prime 2}+3 n^{\prime}-1\right)^{-1 / 2} \delta^{3 / 2},
\end{aligned}
$$

to lowest order in $\delta$. In rectangular coordinates relation (26) becomes

$$
\begin{aligned}
y_{\text {caustic }} / a= & \pm\left(8 / 3^{3 / 2}\right)\left(n^{\prime}-1\right)^{3 / 2} n^{\prime-1 / 2} \\
& \times\left(-n^{\prime 2}+3 n^{\prime}-1\right)^{-1}\left[\left(f-x_{\text {caustic }}\right) / a\right]^{3 / 2} .
\end{aligned}
$$

As a check of both the semiclassical approximation of Eqs. (17) and (18) and the Pearcey approximation of the phase function, the shape of the $p=1$ caustic was also calculated in ray theory by using the procedure of Appen$\operatorname{dix}$ A of Ref. 8 to obtain the envelope of the $p \geqslant 1$ family of scattered rays. The shape of the caustic outside the cylinder for arbitrary $p$ is

$$
\begin{aligned}
x_{\text {caustic }} / a= & (-1)^{p+1}\left[\cos \left(\theta_{i}-2 p \theta_{r}\right)\right. \\
& \left.-R \cos \left(2 \theta_{i}-2 p \theta_{r}\right)\right], \\
y_{\text {caustic }} / a= & (-1)^{p}\left[\sin \left(\theta_{i}-2 p \theta_{r}\right)\right. \\
& \left.-R \sin \left(2 \theta_{i}-2 p \theta_{r}\right)\right],
\end{aligned}
$$

where $\theta_{i}$ is the projection in the $x y$ plane of the angle of incidence of a ray on the cylinder,

$$
\sin \theta_{i}=n^{\prime} \sin \theta_{r},
$$

and

$$
\begin{aligned}
R= & \left(\cos \theta_{i}\right)\left(n^{\prime} \cos \theta_{r}-2 p \cos \theta_{i}\right) \\
& /\left[2\left(n^{\prime} \cos \theta_{r}-p \cos \theta_{i}\right)\right] .
\end{aligned}
$$

The focal line of the $p=1$ near-zone focusing caustic corresponds to $\theta_{i}=0$, and the caustic attaches to the cylinder surface when $R=0$ or

$$
\cos \theta_{i}=\left[\left(n^{\prime 2}-1\right) / 3\right]^{1 / 2} .
$$

The approximate shape of the $p=1$ caustic in the vicinity of the focal line may be determined in ray theory by Taylor-series expanding Eqs. (28)-(30) in powers of $\theta_{i}$. The resulting expression is identical to relation (27) obtained by using the semiclassical approximation. The predictions of ray theory and the semiclassical approximation differ, however, far from the focal line. This should not be surprising, since our semiclassical derivation was meant to accurately model the portion of the caustic in the vicinity of the cusp line. As an illustration of the comparison between ray theory and the semiclassical approximation, the exact $p=1$ near-zone scattered intensity was computed by using Eqs. (4) and (6) for an unpolarized normally incident plane wave, with $\lambda=0.6328 \mu \mathrm{m}, \quad a=10.07 \mu \mathrm{m}$, and $n=1.33$ and is shown in Fig. 2(a). An enlargement of Fig. 2(a) between the cylinder surface and the focal line is reproduced in Fig. 2(b) along with the shape of the $p=1$ caustic obtained by using the semiclassical approximation [Eq. (24)] and ray theory [Eqs. (28)-(30)]. The ray theory result qualitatively agrees with the caustic position expected from the exact wave intensity all the way from the attachment to the cylinder surface to the cusp focal line. The semiclassical approximation result deviates from the expected caustic shape near the attachment. This deviation occurs for two reasons. First, the portion of the

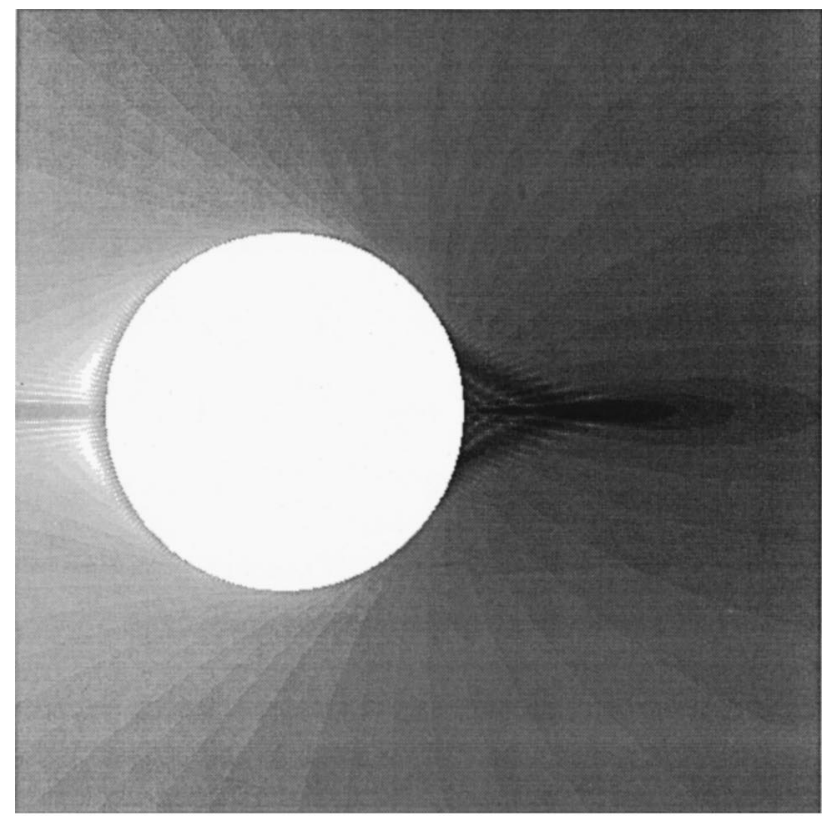

(a)

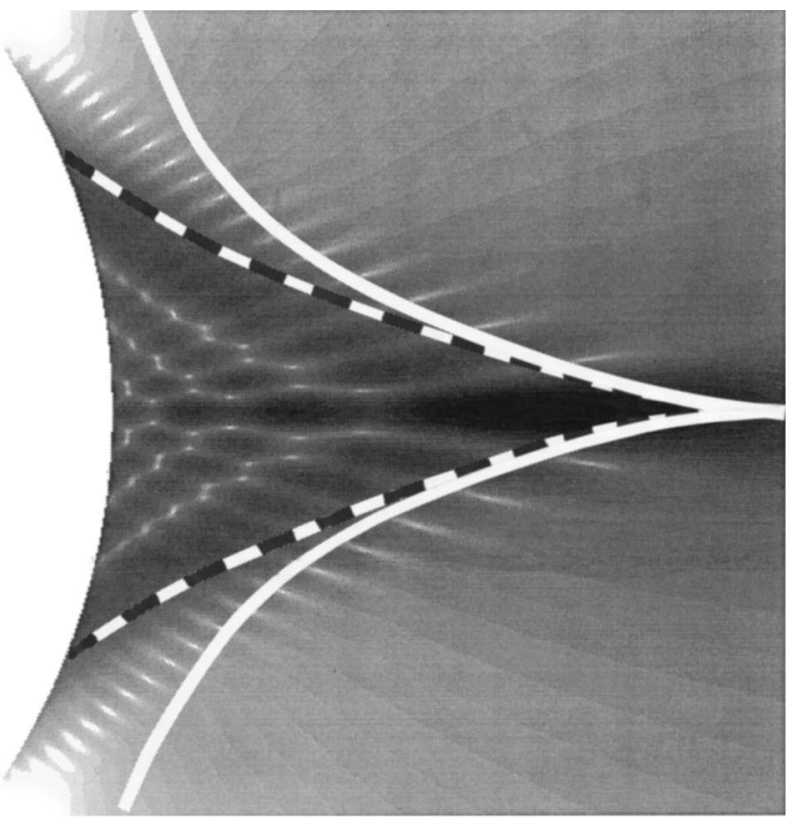

(b)

Fig. 2. (a) $p=1$ portion of the exact near-zone scattered intensity for normal incidence, $\lambda=0.6328 \mu \mathrm{m}, a=10.07 \mu \mathrm{m}$, and $n$ $=1.33$. The shades of black and white correspond to large and small intensities, respectively. The position of the $p=1$ caustic focal line is $r / a=2$. (b) The $p=1$ portion of the exact scattered intensity in the region between the cylinder surface and the caustic focal line. The white and dashed curves are the position of the $p=1$ caustic as predicted by the semiclassical approximation and ray theory, respectively. 
caustic near the attachment corresponds to $l \approx x$ or $w$ $\approx 1$, where the Taylor-series expansion of the phase function of Eq. (17) in powers of $w$ is very slowly convergent. In addition, the asymptotic expansions of the Hankel functions of relations (9) lose their validity for the combined conditions ${ }^{27} l \approx x$ and $r \approx a$, which correspond to the attachment region.

\section{SHAPE OF THE $p \geqslant 2$ CAUSTICS}

A. $p \geqslant 2$ Rainbows in the Near Zone

The two curved sheets of the rainbow caustic are qualitatively illustrated in Fig. 3. For $p \geqslant 2$ the scattering angle of Eq. (20) reaches an extremum corresponding to the $(p-1)$-order rainbow a distance $r$ from the cylinder axis when

$$
\begin{aligned}
\mathrm{d} \theta / \mathrm{d} w=0 & =\left(1-w_{r}^{2}\right)^{-1 / 2}-\left(p / n^{\prime}\right)\left(1-w_{r}{ }^{2} / n^{\prime 2}\right)^{-1 / 2} \\
& -[1 /(2 r / a)]\left[1-w_{r}{ }^{2} /(r / a)^{2}\right]^{-1 / 2}
\end{aligned}
$$

The $r \rightarrow \infty$ limit of the rainbow impact parameter $w_{r}$ of Eq. (32) is

$$
w_{r}=\left[\left(p^{2}-n^{\prime 2}\right) /\left(p^{2}-1\right)\right]^{1 / 2},
$$

which agrees with ray theory. ${ }^{28,29}$ The scattering angle of the $(p-1)$-order rainbow a distance $r$ from the cylinder axis is obtained by substituting the solution of Eq. (32) for $w_{r}$ into Eq. (20), giving

$$
\begin{aligned}
\theta_{\text {rainbow }}(r / a)= & (p-1) \pi+2 \arcsin w_{r} \\
& -2 p \arcsin \left(w_{r} / n^{\prime}\right)-\arcsin \left[w_{r} /(r / a)\right] .
\end{aligned}
$$

We now derive the periodicity of the supernumerary structure associated with the $(p-1)$-order rainbow for any value of $r / a$. For scattering angles near the $(p-1)$-order rainbow, we define $\varepsilon$ and $\Delta$ by

$$
\begin{aligned}
w & =w_{r}+\varepsilon, \\
\theta & =\theta_{\text {rainbow }}+\Delta .
\end{aligned}
$$

We Taylor-series expand the phase $\Phi(w)$ of Eq. (17) about $w_{r}$ in powers of $\varepsilon$ and retain terms of order $\varepsilon^{3}$ or less so as to include the two stationary points of $\Phi(w)$ (i.e., the two supernumerary rays) near $w_{r}$. The scattered fields in the vicinity of the rainbow caustic are then proportional to the Airy integral of Eq. (A1), which we write as

$$
E_{\text {scatt }} \propto c B_{\text {scatt }} \propto A i\left(-x^{2 / 3} \Delta / h^{1 / 3}\right) .
$$

The periodicity of the supernumerary structure, parameterized by $h$, is then

$$
\begin{gathered}
h(r / a)=w_{r}\left(1-w_{r}{ }^{2}\right)^{-3 / 2}-\left(p w_{r} / n^{\prime 3}\right)\left(1-w_{r}{ }^{2} / n^{\prime 2}\right)^{-3 / 2} \\
-\left(w_{r} / 2\right)(r / a)^{-3}\left[1-w_{r}{ }^{2} /(r / a)^{2}\right]^{-3 / 2} .
\end{gathered}
$$

The $r \rightarrow \infty$ limit of Eq. (38) is

$$
h=\left(p^{2}-1\right)^{2}\left(p^{2}-n^{\prime 2}\right)^{1 / 2} /\left[p^{2}\left(n^{\prime 2}-1\right)^{3 / 2}\right],
$$

which agrees with Airy theory. ${ }^{15,30}$

The predictions of Eqs. (32), (34), and (38) were tested as follows. Equation (32) was numerically solved for $w_{r}$ for a number of values of $r / a$ for $p=2$ and $n^{\prime}=1.5$. The result was then substituted into Eqs. (34) and (38) to obtain $\theta_{\text {rainbow }}(r / a)$ and $h(r / a)$, which are shown as the solid curve in Figs. 4 and 5, respectively. The dashed curves are the far-zone limits $\theta_{\text {rainbow }}(\infty)=157.1585^{\circ}$ and $h(\infty)=2.1298$. The rainbow angle in the near zone was also computed by using ray theory through Eqs. (28)-(30) and is shown as the set of triangles in Fig. 4. In addition, the $p=2$ Debye portion of the exact scattered intensity was computed for various values of $r / a$ for the conditions of normal incidence, $n=1.5$, and $k a=1000$. We assumed that the $p=2$ Debye intensity is proportional to $\operatorname{Ai}^{2}\left[-x^{2 / 3}\left(\theta-\theta_{\text {rainbow }}\right) / h^{1 / 3}\right]$, in accordance with relation (37). For each value of $r / a$, the rainbow angle $\theta_{\text {rainbow }}$

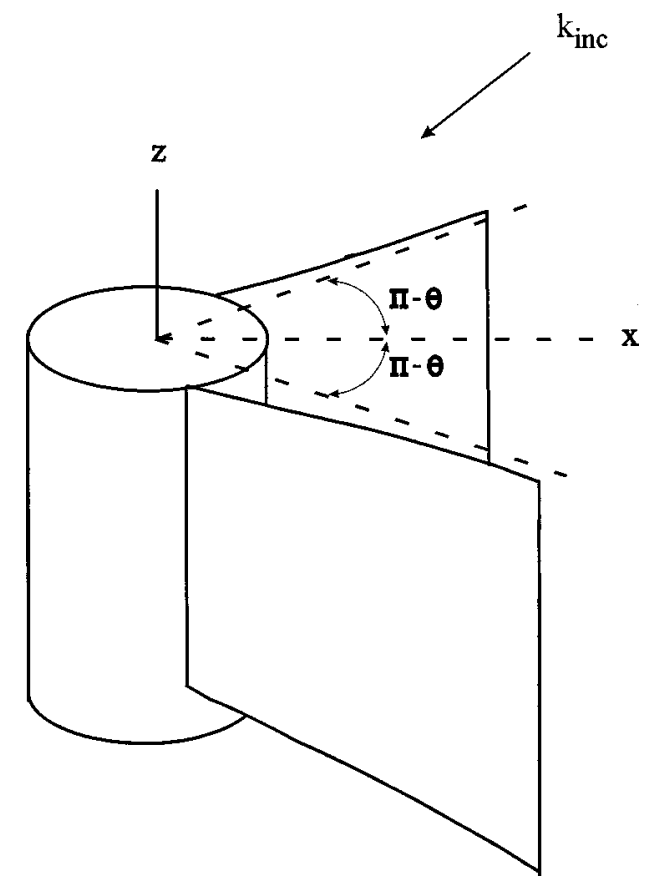

Fig. 3. $p=2$ rainbow caustic for a plane wave whose angle of incidence is less than that of the caustic merging transition.

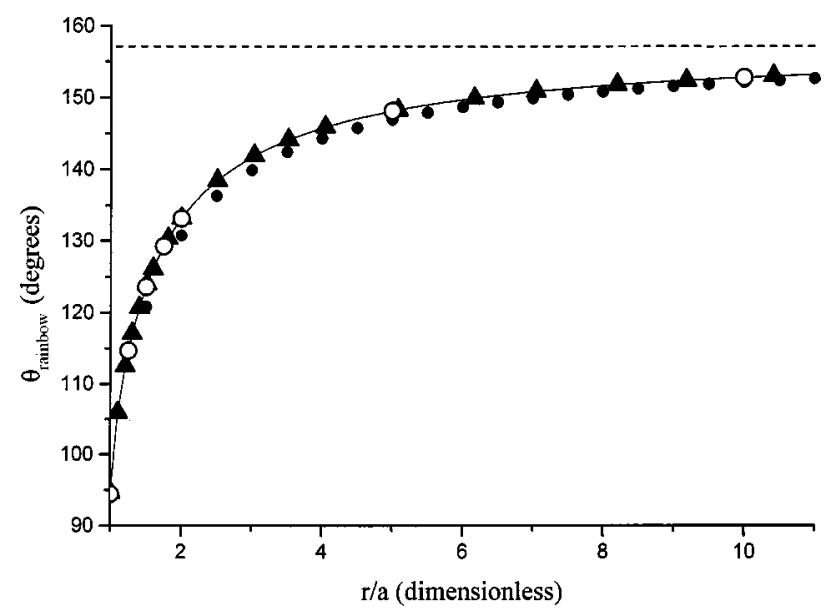

Fig. 4. $p=2$ rainbow angle $\theta_{\text {rainbow }}$ as a function of $r / a$, as predicted by the semiclassical approximation (solid curve), and the asymptotic $r \rightarrow \infty$ rainbow angle $\theta_{\text {rainbow }}(\infty)$ (dashed line) for $n^{\prime}$ $=1.5$. The prediction of ray theory is the set of triangles, the prediction of exact electromagnetic wave theory fitted to the square of an Airy integral is the set of open circles, and a straight-line ray trajectory at the angle $\theta_{\text {rainbow }}(\infty)$ is the set of solid circles. 


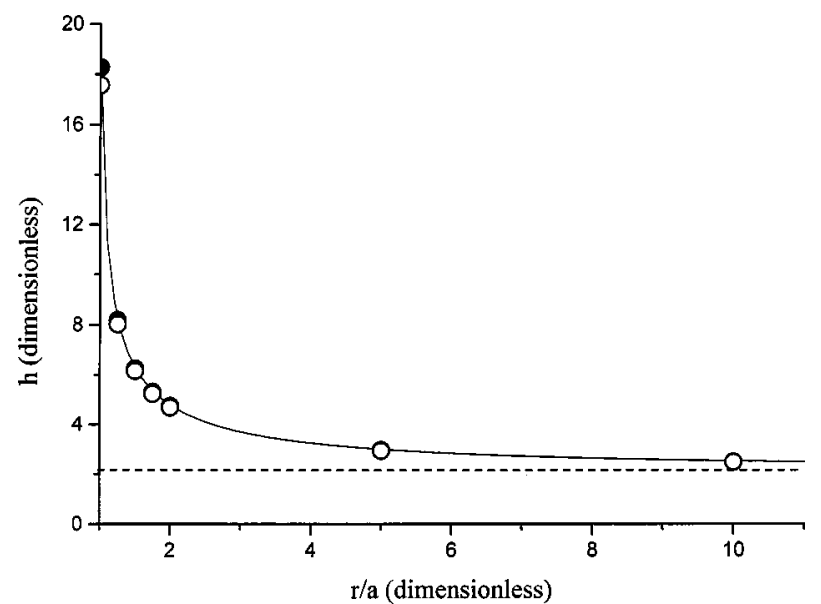

Fig. 5. Supernumerary periodicity parameter $h$ as predicted by the semiclassical approximation as a function of $r / a$ for $n^{\prime}$ $=1.5$. The prediction of exact electromagnetic wave theory fitted to the square of an Airy integral by using the first and second Airy peaks and the first and third Airy peaks is given by the set of solid and open circles, respectively. Dashed line as in Fig. 4.

was determined from the position of the first Airy maximum by using the results of Appendix A and is shown as the set of open circles in Fig. 4. The value of $h$ was determined from the spacing of the first and second Airy maxima, and from that of the first and third Airy maxima, and is shown in Fig. 5 as the solid and open circles, respectively. As is seen in the two figures, the predictions of the semiclassical approximation for $\theta_{\text {rainbow }}(r / a)$ and $h(r / a)$ agree quite closely with those of both ray theory and exact electromagnetic wave scattering all the way from the cylinder surface to the far zone. The reason that the semiclassical approximation accurately describes the $p \geqslant 2$ rainbow caustics near the attachment, whereas it poorly describes the $p=1$ focusing caustic there, is that the reference point $w_{0}$ of the Taylorseries expansion of the phase function for the rainbow caustics continually shifts along the caustic as $r$ is varied. For the focusing caustic, on the other hand, the reference point did not shift along the caustic as $r$ is varied, but remained at $w_{0}=0$.

The straight-line ray trajectory at the angle $\theta_{\text {rainbow }}(\infty)$ extending from the attachment of the rainbow ray at the cylinder surface to the far zone is also shown in Fig. 4 as the set of solid circles. The fact that $\theta_{\text {rainbow }}$ does not coincide with the straight-line ray trajectory illustrates the curvature of the rainbow caustic in the near zone. Both $\theta_{\text {rainbow }}(r / a)$ and $h(r / a)$ approach $\theta_{\text {rainbow }}(\infty)$ and $h(\infty)$ quite slowly. For $r / a=10,50,100$, and 250, the difference between $\theta_{\text {rainbow }}(r / a)$ and $\theta_{\text {rainbow }}(\infty)$ is $4.44^{\circ}, 0.88^{\circ}$, $0.44^{\circ}$, and $0.18^{\circ}$, respectively, and the difference between $h(r / a)$ and $h(\infty)$ is $19.98 \%, 3.87 \%, 1.93 \%$, and $0.77 \%$. Thus, for the detector array to be in the far zone for rainbow refractometry experiments, $r / a$ must be of the order of at least a few hundred. The near-zone behavior of the rainbow angle and the supernumerary spacing were also computed for a number of other values of $n^{\prime}$ and $p$. For fixed $p$ we found that $\theta_{\text {rainbow }}$ and $h$ in the near zone deviated from their respective asymptotic values more for $n^{\prime} \approx p$ than for $n^{\prime} \ll p$. For fixed $n^{\prime}$ they deviated from their asymptotic values more for small $p$ than for large $p$.
B. $p \geqslant 2$ Caustic Merging Transition at $r \rightarrow \infty$

As $n^{\prime}$ increases by tilting the incident plane wave at a steeper angle, the two sheets of the $(p-1)$-order rainbow caustic of Fig. 3 migrate toward either $\theta=0$ or $\theta=\pi$ as $r \rightarrow \infty$. When $n^{\prime}=p$, the two sheets meet in a cusp focal line at infinity, ${ }^{31}$ as is suggested in Fig. 6. At this transition the conditions $r \rightarrow \infty$ and $p=n^{\prime}$ applied to Eq. (32) require that $w_{r}=0$, i.e., constructive interference of paraxial rays dominates the merging of the two caustic sheets at infinity.

We now examine the properties of the $(p-1)$-order rainbow caustic in the far zone for values of $n^{\prime}$ slightly smaller than $p$, where the two rainbow sheets do not quite join. Since paraxial rays dominate the rainbow caustic in the far zone for $n^{\prime} \approx p$, we expand the phase $\Phi(w)$ of Eq. (17) for $r \rightarrow \infty$ in powers of $w$ and retain the terms of order $w^{4}$ or less so as to include the two stationary points of the two supernumerary rays, as well as that of the third ray incident on the other side of the cylinder axis that interferes with the two supernumerary rays when

$$
n^{\prime}>[\sin (\pi / 2 p)]^{-1} \text {. }
$$

When $\Phi(w)$ is truncated at order $w^{4}$, the $r \rightarrow \infty$ scattered fields as a function of $\theta$ and $n^{\prime}$ near the caustic merging transition are proportional to the Pearcey integral of Eq. (A2) with

$$
\begin{aligned}
& X=\left(12 n^{\prime} x\right)^{1 / 2}\left(p-n^{\prime}\right) /\left(n^{\prime 3}-p\right)^{1 / 2}, \\
& Y=(12)^{1 / 4}\left(n^{\prime} x\right)^{3 / 4}[\theta-(p-1) \pi] /\left(n^{\prime 3}-p\right)^{1 / 4} .
\end{aligned}
$$

The far-zone scattering angle of the $(p-1)$-order rainbow for $n^{\prime} \approx p$ is given by Eq. (A3), or

$$
\theta_{\text {rainbow }}=(p-1) \pi \pm\left(2^{5 / 2} / 3\right)\left(p-n^{\prime}\right)^{3 / 2} /\left(n^{\prime 3}-p\right)^{1 / 2} \text {. }
$$

The $\theta$-dependence of the far-zone scattered fields in the vicinity of the caustic merging transition is given by a cross section through the Pearcey integral of Eqs. (41) and (A2) when graphed as a function of $\theta$ and $n^{\prime}$. The rainbow angle as a function of $n^{\prime}$ is the caustic ridge of the Pearcey integral and is shown in Fig. 7. For $n^{\prime}<p$ the

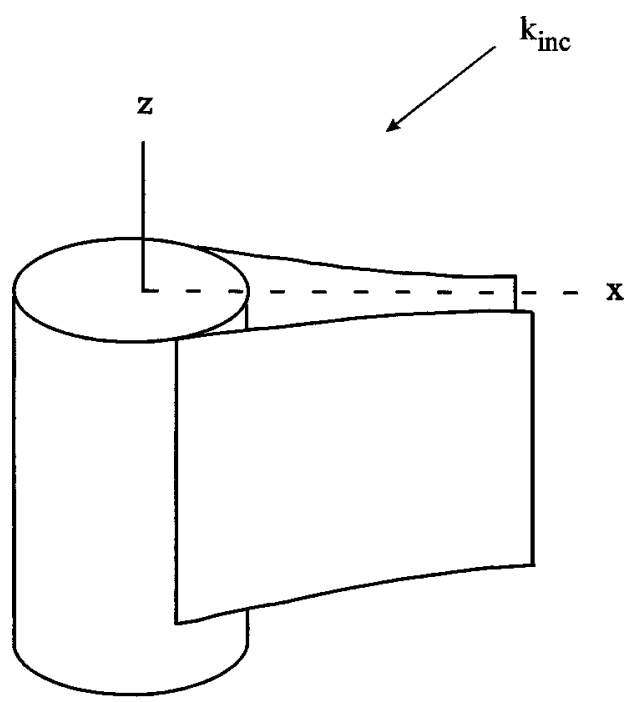

Fig. 6. $p=2$ rainbow caustic for a plane wave whose angle of incidence is equal to that of the caustic merging transition. 


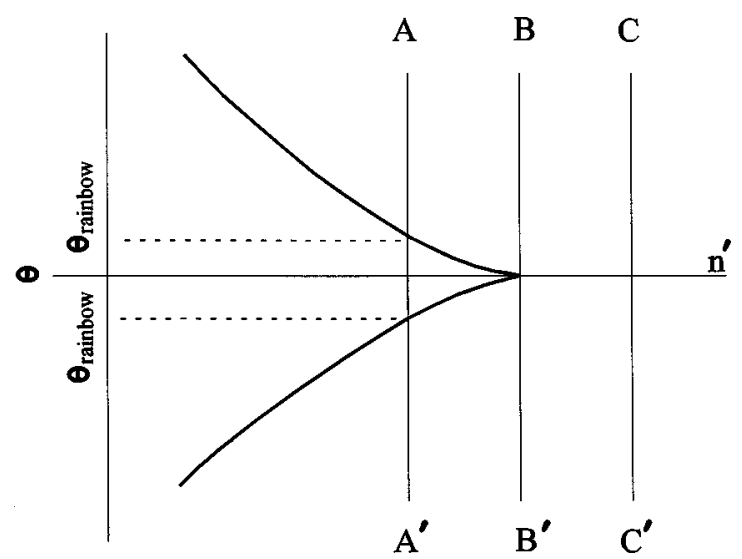

Fig. 7. ( $p-1)$-order rainbow angle as a function of $n^{\prime}$ in the vicinity of the caustic merging transition. The sections $A A^{\prime}$, $B B^{\prime}$, and $C C^{\prime}$ correspond to the plane-wave angle of incidence less than, equal to, and greater than that of the transition.

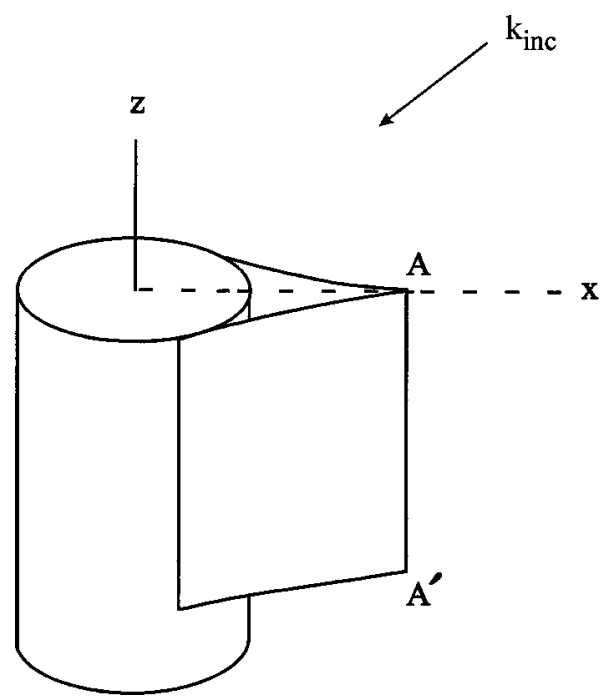

Fig. 8. $p=2$ rainbow caustic for a plane wave whose angle of incidence is greater than that of the caustic merging transition and less than that of the exterior/interior cusp transition. The caustic focal line is $A A^{\prime}$.

cross section $A A^{\prime}$ cuts the caustic ridge twice, giving the rainbow and its supernumerary interference pattern, as is described in Appendix A [see also Fig. 4(a) of Ref. 4]. For $n^{\prime}=p$ the cross section $B B^{\prime}$ passes through the cusp point corresponding to the field enhancement at the merging of the two sheets of the rainbow caustic at infinity (see Fig. 4(c) of Ref. 4). For $n^{\prime}>p$ the cross section $C C^{\prime}$ lies to the right of the cusp point. As a result, the $(p-1)$-order rainbow no longer propagates all the way out to infinity; rather, it becomes a near-zone caustic, as is qualitatively illustrated in Fig. 8, and the far-zone scattered fields are slowly varying in $\theta$ [see Fig. 4(d) of Ref. 4]. This interpretation of Fig. 7 for $n^{\prime}<p$ may be demonstrated as follows. In the far zone, the rainbow angle $\theta_{\text {rainbow }}$ is given by Eq. (34) with $w_{r}$ given by Eq. (33). The scattered electric field is given by relation (37) with $h$ given by Eq. (39). On the other hand, the rainbow angle as predicted by the Pearcey approximation (called $\theta_{\text {Pearcey }}$ for the remainder of this subsection) is given by Eq. (42), and a cross section through the Pearcey integral is given by Eq. (A4) and relation (A5) with $X$ and $Y$ given by Eqs. (41). When $n^{\prime} \ll p, \theta_{\text {rainbow }}$ and $\theta_{\text {Pearcey }}$ differ from each other, as do the arguments of the respective Airy integrals. But when

$$
n^{\prime}=p-\varepsilon,
$$

with $\varepsilon$ being small, the rainbow and Pearcey approximation scattering angles become

$$
\begin{aligned}
\theta_{\text {rainbow }}= & (p-1) \pi-2^{5 / 3} \varepsilon^{3 / 2} /\left[3 p^{1 / 2}\left(p^{2}-1\right)^{1 / 2}\right] \\
& -2^{1 / 2}\left(7 p^{2}-3\right) \varepsilon^{5 / 2} /\left[5 p^{3 / 2}\left(p^{2}-1\right)^{3 / 2}\right]+\cdots, \\
\theta_{\text {Pearcey }}= & (p-1) \pi-2^{5 / 3} \varepsilon^{3 / 2} /\left[3 p^{1 / 2}\left(p^{2}-1\right)^{1 / 2}\right] \\
& -2^{3 / 2} p^{1 / 2} \varepsilon^{5 / 2} /\left(p^{2}-1\right)^{3 / 2}+\cdots,
\end{aligned}
$$

and the electric field in the vicinity of the caustics is

$$
\begin{aligned}
E_{\text {rainbow }} \propto & \operatorname{Ai}\left\{-x^{2 / 3} p^{1 / 2}\right. \\
& \left.\times\left(\theta-\theta_{\text {rainbow }}\right) /\left[2^{1 / 6}\left(p^{2}-1\right)^{1 / 6} \varepsilon^{1 / 6}\right]\right\}, \\
E_{\text {Pearcey }} \propto & \operatorname{Ai}\left\{-x^{2 / 3} p^{1 / 2}\right. \\
& \left.\times\left(\theta-\theta_{\text {Pearcey }}\right) /\left[2^{1 / 6}\left(p^{2}-1\right)^{1 / 6} \varepsilon^{1 / 6}\right]\right\} .
\end{aligned}
$$

The two sets of expressions are identical to first order in $\varepsilon$.

In Ref. 6 the $p=2$ rainbow angle in the vicinity of the caustic merging transition was modeled as

$$
\theta_{\text {rainbow }}=\pi \pm K^{1 / 2}\left(\xi_{c}-\xi\right)^{3 / 2}
$$

on the basis of generalized ray-tracing arguments, where $\xi_{c}$ is the tilt angle of the incident beam at the transition. For $p=2, n=1.49$, and $\xi_{c}=50.37^{\circ}$, corresponding to a poly(methyl methocrylate) plastic fiber, and for $p=2$, $n=1.31$, and $\xi_{c}=60.75^{\circ}$, corresponding to an ice cylinder, Marston ${ }^{6}$ numerically computed the first-order rainbow scattering angle by using Eqs. (33) and (34) with $r \rightarrow \infty$ as a function of $\xi$ and numerically fitted the results near $\xi_{c}$ to Eq. (46). His fitting procedure obtained $K=0.06148 / \mathrm{deg}$ and $K=0.1988 / \mathrm{deg}$, respectively. To test these results, we parameterize $n^{\prime}$ by Eq. (43) and let

$$
\xi=\xi_{c}-\delta
$$

near the transition. Substituting Eqs. (43) and (47) into Eq. (3) gives

$\varepsilon=\delta\left(p^{2}-1\right)\left(p^{2}-n^{2}\right)^{1 / 2} /\left[p\left(n^{2}-1\right)^{1 / 2}\right]+O\left(\delta^{2}\right)$,

and substituting Eq. (48) into Eq. (42) gives

$$
K=32\left(p^{2}-1\right)^{2}\left(p^{2}-n^{2}\right)^{3 / 2} /\left[9 p^{4}\left(n^{2}-1\right)^{3 / 2}\right] .
$$

Evaluating Eq. (49) for $n=1.49$ and $n=1.31$ with $p=2$ gives $K=0.06150 / \mathrm{deg}$ and $K=0.19882 / \mathrm{deg}$, respectively, in agreement with the numerical results of Ref. 6.

Our experimental observation of the $p=2$ caustic merging transition is shown in Figs. 9(a), 9(b), and 9(c) for $\xi=\xi_{c}-3^{\circ}, \xi=\xi_{c}$, and $\xi=\xi_{c}+8^{\circ}$, respectively. The beam of a $3-\mathrm{mW} \mathrm{He}-\mathrm{Ne}$ laser was expanded to a diameter of $25.4 \mathrm{~mm}$ and was incident on a 13.4-cm-long glass rod whose diameter was nominally $15.9 \mathrm{~mm}$ and whose refractive index was previously measured to be $n=1.474$ \pm 0.002. The rod's cross section was slightly elliptical, 


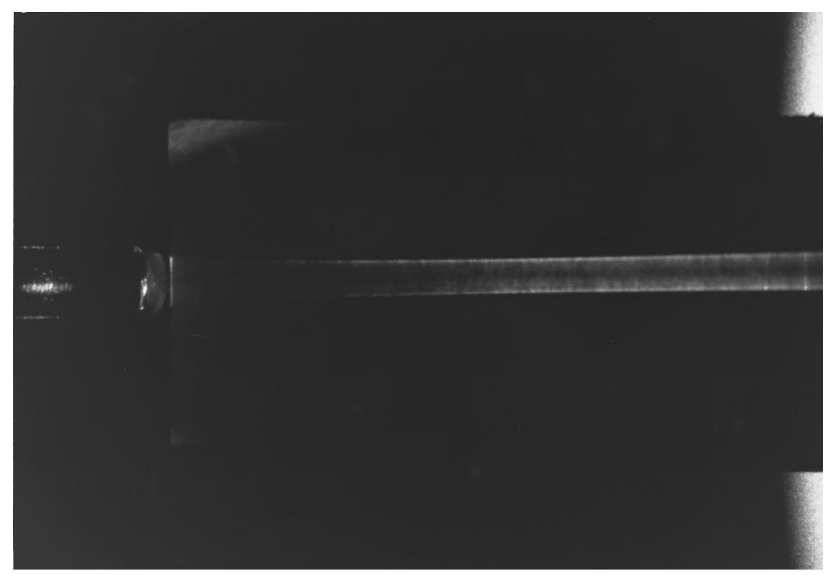

(a)

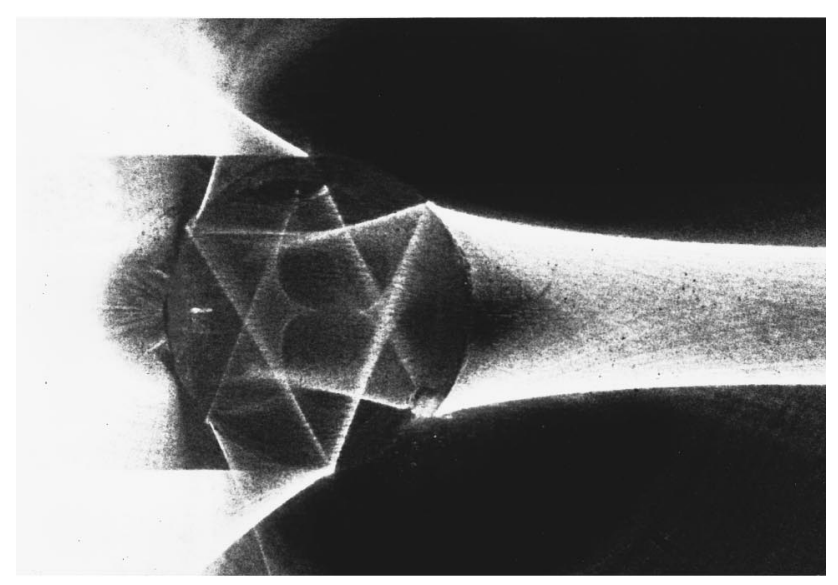

(b)

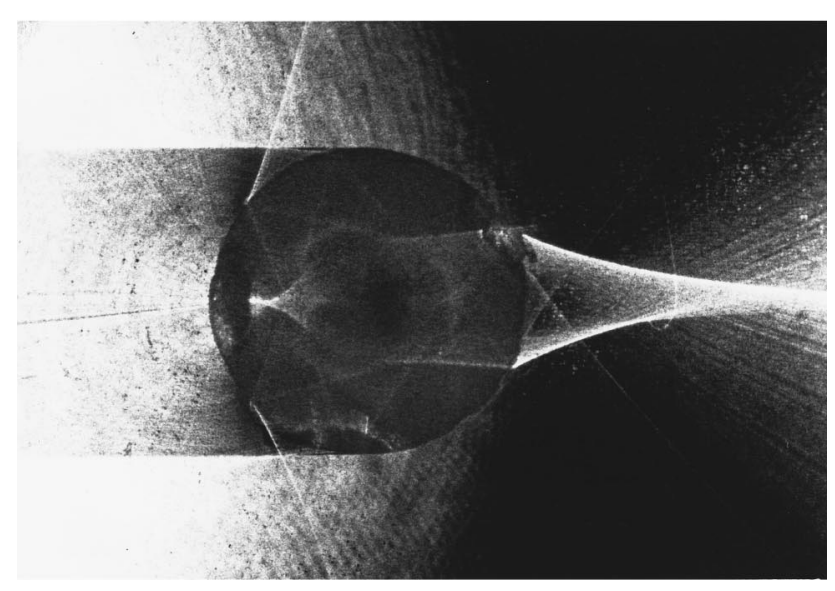

(c)

Fig. 9. $\quad p=2$ caustic merging transition for a nominally 15.9$\mathrm{mm}$-diameter glass rod with refractive index 1.474 and illuminated by a plane wave with tilt angles (a) $\xi=\xi_{c}-3^{\circ}$, (b) $\xi=\xi_{c}$, and (c) $\xi=\xi_{c}+8^{\circ}$ with $\xi_{c}=46.58^{\circ} \pm 0.08^{\circ}$. In (a) the beam is incident from the right, the rod is at the left, and the caustic illuminates a white piece of cardboard placed perpendicular to the axis of the rod at its base. The rainbow caustic has a far-zone scattering angle $\theta_{\text {rainbow }}(\infty) \approx 178.5^{\circ}$. In (b) and (c), the beam is incident from the right, the dark region to the left of the rod is its shadow, and the exterior and interior caustics illuminate a piece of tissue paper held firmly in a frame perpendicular to the axis of the rod at its base. with the minor-axis-to-major-axis ratio being $a / b$ $=0.962 \pm 0.001$. The $p=2$ caustic merging transition occurs when the $p=1$ interior paraxial rays strike the cylinder surface at the $x$ axis. If the cross section of the rod is circular, this occurs when

$$
\cos \xi_{c}=\left[\left(n^{2}-1\right) / 3\right]^{1 / 2} .
$$

But for an elliptical-cross-section rod with the longer axis of the ellipse being along the $x$ axis, we obtain rather ${ }^{3}$

$$
\cos \xi_{c}=\left(n^{2}-1\right)^{1 / 2}\left[2(b / a)^{2}-1\right] /\left[4(b / a)^{2}-1\right]^{1 / 2} \text {. }
$$

Experimentally, we found that $\xi_{c}=46.58^{\circ} \pm 0.08^{\circ}$. Substitution of $n=1.474$ and $b / a=1.0395$ into Eq. (51) gives $\xi_{c}=46.382^{\circ}$, in agreement with our experimental result. The change in $\xi_{c}$ due to the roughly $4 \%$ elipticity of the rod is substantial, since substitution of $n=1.474$ into Eq. (50) for a circular cross section gives $\xi_{c}$ $=51.302^{\circ}$.

\section{C. $p \geqslant 2$ Near-Zone Cusp Caustics}

When the tilt angle of the diagonally incident plane wave is increased further, so that $p<n^{\prime}<2 p$, the scattered fields in the vicinity of the $p \geqslant 2$ near-zone focusing caustic are obtained by assuming that $w$ is small (corresponding to paraxial rays), expanding the phase of Eq. (17) in powers of $w$, and truncating the expansion at $w^{4}$. The resulting Pearcey integral of Eq. (A2) is described by

$$
\begin{aligned}
& X=(24 k r \cos \xi)^{1 / 2}\left[\left(n^{\prime}-p\right) / n^{\prime}\right][(f-r) / a] / D^{1 / 2}, \\
& Y=(24)^{1 / 4}(k r \cos \xi)^{3 / 4}[\theta-(p-1) \pi] / D^{1 / 4}
\end{aligned}
$$

where

$$
\begin{aligned}
D & =\left[2(r / a)^{3}\left(n^{\prime 3}-p\right) / n^{\prime 3}\right]-1, \\
f / a & =n^{\prime} /\left[2\left(n^{\prime}-p\right)\right] .
\end{aligned}
$$

The cusp focal line moves in from infinity when $n^{\prime}=p$, and the exterior/interior cusp transition occurs at $n^{\prime}$ $=2 p$. The shape of the near-zone focusing caustic in the Pearcey approximation is given by Eq. (A3), or

$$
\begin{aligned}
\theta_{\text {caustic }}(r)= & (p-1) \pi \pm(8 / 3)\left[\left(n^{\prime}-p\right) / n^{\prime}\right]^{3 / 2} \\
& \times\left[\left(f-r_{\text {caustic }}\right) / a\right]^{3 / 2} / D^{1 / 2} .
\end{aligned}
$$

In the vicinity of the focal line, we may write

$$
r_{\text {caustic }} / a=f / a-\delta
$$

and expand Eq. (55) in powers of $\delta$. Near the cusp line, we obtain

$$
\begin{aligned}
\theta_{\text {caustic }} \approx & (p-1) \pi \pm(16 / 3)\left(n^{\prime}-p\right)^{3} n^{\prime-3 / 2} \\
& \times\left[4\left(n^{\prime}-p\right)^{3}-n^{\prime 3}+p\right]^{-1 / 2} \delta^{3 / 2},
\end{aligned}
$$

to lowest order in $\delta$. For $n^{\prime}>2 p$ the cusp caustic lies entirely inside the cylinder, as was the case for the $p=1$ caustic $^{3}$ for $n^{\prime}>2$. For $p=1$, Eqs. (52), (53), (54), and (55) and relation (57) reduce to Eqs. (21), (22), (23), and (24) and relation (26), respectively.

Our experimental observation of the exterior/interior cusp transition of the $p=2$ caustic is shown in Figs. 10(a), 10(b), and 10(c), corresponding to a plane-wave tilt angle below, at, and above the transition, respectively. Figure 10 is qualitatively identical to the external/ internal cusp transition of the $p=1$ caustic illustrated in 


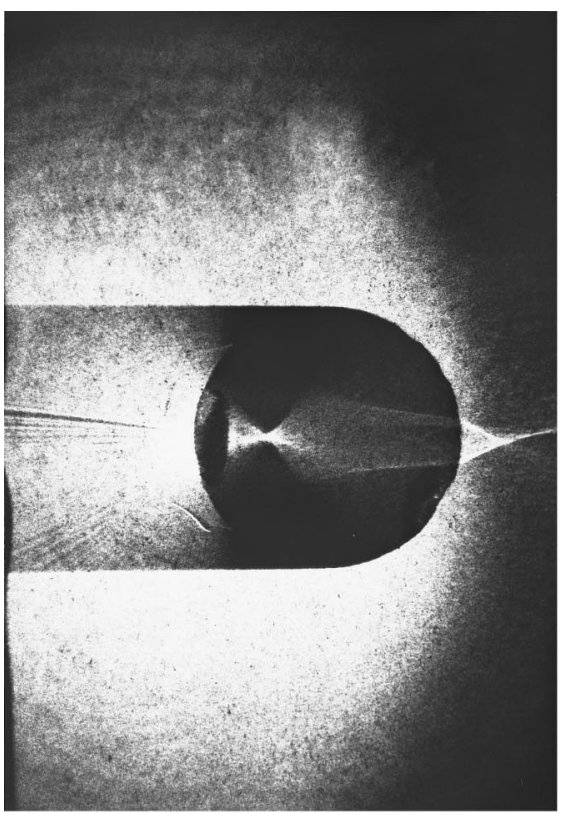

(a)

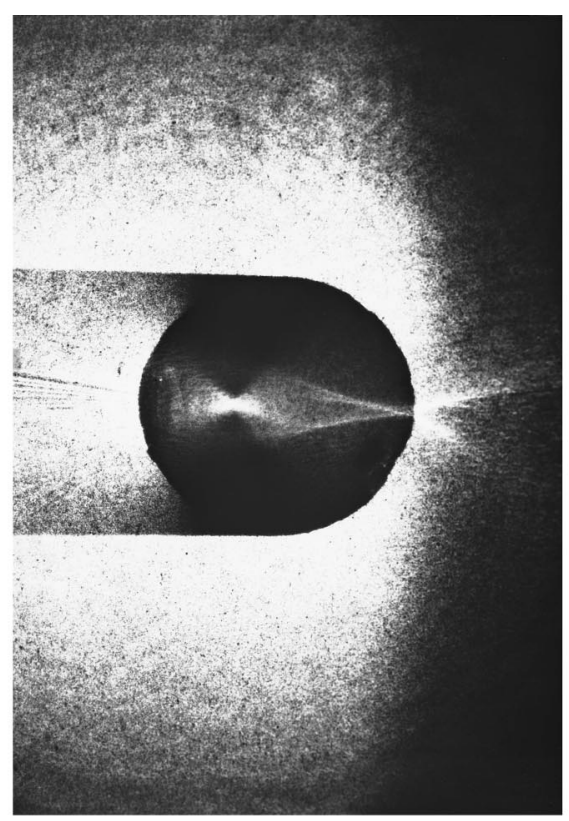

(b)

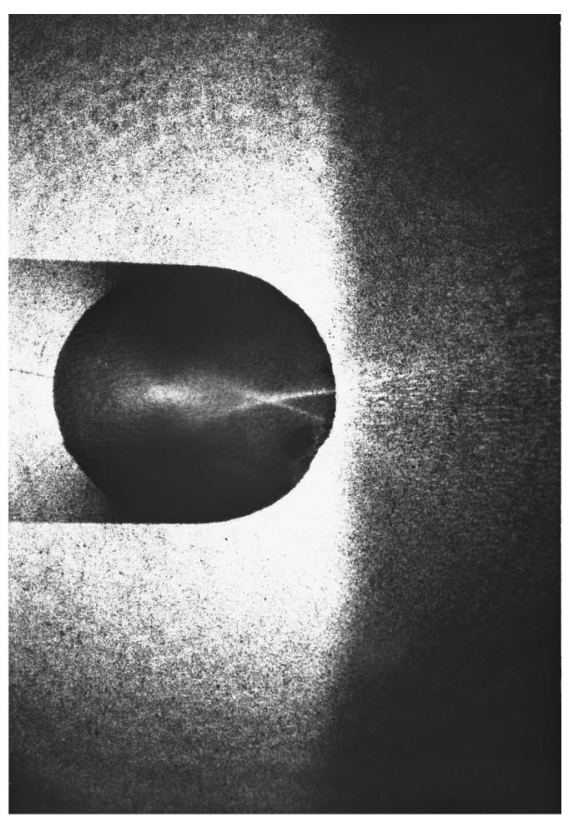

(c)

Fig. 10. $p=2$ exterior/interior cusp caustic transition for a nominally 15.9-mm-diameter glass rod with refractive index 1.474 and illuminated by a plane wave with the tilt angles (a) $\xi<\xi_{c^{\prime}}$, (b) $\xi=\xi_{c^{\prime}}$, and (c) $\xi>\xi_{c^{\prime}}$ with $\xi_{c^{\prime}}=67.83^{\circ} \pm 0.08^{\circ}$. The beam is incident from the right, and the caustics are observed by using the method described in Figs. 9(b) and 9(c).

ray theory in Fig. 3 of Ref. 3. The transition for $p=2$ occurs when the $p=2$ interior paraxial rays strike the cylinder surface at the $x$ axis. For a circular-crosssection rod, this occurs when

$$
\cos \xi_{c^{\prime}}=\left[\left(n^{2}-1\right) / 15\right]^{1 / 2}
$$

For an elliptical-cross-section rod oriented with the long axis of the ellipse along the $x$ axis, it occurs when

$$
\begin{aligned}
\cos \xi_{c^{\prime}}= & \left(n^{2}-1\right)^{1 / 2}\left[8(b / a)^{4}-8(b / a)^{2}+1\right] / \\
& {\left[64(b / a)^{6}-64(b / a)^{4}+16(b / a)^{2}-1\right]^{1 / 2} . }
\end{aligned}
$$

Experimentally, we found that $\xi_{c^{\prime}}=67.83^{\circ} \pm 0.08^{\circ}$. Again, substitution of $n=1.474$ and $b / a=1.0395$ into Eq. (59) gives $\xi_{c^{\prime}}=67.110^{\circ}$, in good agreement with the experimental result. As was the case for the caustic merging transition in Subsection 4.B, the angle of the second transition sensitively depends on the rod's ellipticity, since substitution of $n=1.474$ into Eq. (58) for a circular cross section gives $\xi_{c^{\prime}}=73.763^{\circ}$.

\section{DISCUSSION}

In the context of catastrophe theory, the complete morphology of the $p$-caustic of the waves scattered by a highsymmetry particle is a function of a number of control parameters, including the particle's eccentricity, its refractive index, the radius of curvature of the phase fronts of the incident beam, the tilt angle of the incident beam, etc. As one or more control parameters are varied, any apparent change of type of the caustic is merely a shifting from one cross section of the full caustic morphology to another in the multidimensional space of the control parameters. For a few high-symmetry particle shapes, one may analytically calculate the full caustic structure as a function of the control parameters, as was done here in Sections 3 and 4 for scattering of a diagonally incident plane wave by a circular cylinder. The semiclassical approximation is applicable as well, with a number of relatively minor changes, to the rainbow caustics of a sphere. Since the exact partial-wave solution to the electromagnetic boundary-value problem is known for scattering by a spheroid and by an elliptical cylinder, it is of interest to determine whether the version of the semiclassical approximation employed here can obtain the full caustic morphology for these lower-symmetry particles.

\section{APPENDIX A: AIRY AND PEARCEY CAUSTICS}

The fold optical caustic is a two-ray to zero-ray transition. The caustic, along with its associated diffraction structure, is described by the Airy integral,${ }^{18}$ which is defined as

$$
\operatorname{Ai}(X) \equiv\left[3^{1 / 3} /(2 \pi)\right] \int_{-\infty}^{\infty} \mathrm{d} u \exp \left[i\left(u^{3}+3^{1 / 3} X u\right)\right] .
$$

The caustic position is $X=0$, and the first three extrema of the supernumerary diffraction structure occur at $X=-1.0188,-3.2482$, and -4.8201 .

The cusp optical caustic is a three-ray to one-ray transition. The caustic, along with its associated diffraction structure, is described by the Pearcey integral, ${ }^{19}$ which is defined as

$$
P(X, Y) \equiv \int_{-\infty}^{\infty} \mathrm{d} u \exp \left[i\left(u^{4}+X u^{2}+Y u\right)\right] .
$$

When graphed with $Y$ as a function of $X$, the shape of the cusp caustic is

$$
Y= \pm(-2 X / 3)^{3 / 2} .
$$


Any cross section through either of the two branches of the caustic away from the cusp point is a fold caustic modulated by the amplitude of the third ray. In particular, for a straight vertical cross section through one of the branches, i.e.,

$$
Y=(-2 X / 3)^{3 / 2}-\Delta
$$

as a function of $\Delta$, we have

$$
\begin{aligned}
P(X, Y)= & 2^{1 / 2} \pi(3|X|)^{-1 / 6} \exp \left[i X^{2} / 12-i(\Delta / 2)(2|X| / 3)^{1 / 2}\right] \\
& \times \operatorname{Ai}\left[-\Delta(24|X|)^{-1 / 6}\right]+[\pi /(3|X|)]^{1 / 2} \\
& \times \exp \left(-2 i X^{2} / 3-i \pi / 4\right),
\end{aligned}
$$

assuming that $X<0$ and $\Delta^{1 / 2} /|X|^{3 / 4} \ll 1$. The first term of relation (A5) is the fold caustic, and the second term is the amplitude of the third participating ray evaluated in the stationary phase approximation.

\section{ACKNOWLEDGMENT}

This work was supported in part by NASA grants NCC3521 and NAG3-2232.

Address correspondence to James A. Lock at the location on the title page or by e-mail, jimandcarol @stratos.net.

\section{REFERENCES AND NOTES}

1. M. V. Berry and C. Upstill, "Catastrophe optics: morphologies of caustics and their diffraction patterns," Prog. Opt. 18, 257-346 (1980); in particular, Sect. 3, pp. 277-297.

2. P. L. Marston, "Geometrical and catastrophe methods in scattering," Phys. Acoust. 21, 1-234 (1992); in particular, Sect. 4.8, Fig. 76, p. 187.

3. C. L. Adler, J. A. Lock, B. R. Stone, and C. J. Garcia, "Highorder interior caustics produced in scattering of a diagonally incident plane wave by a circular cylinder," J. Opt. Soc. Am. A 14, 1305-1315 (1997)

4. J. A. Lock and C. L. Adler, "Debye-series analysis of the first order rainbow produced in scattering of a diagonally incident plane wave by a circular cylinder," J. Opt. Soc. Am. A 14, 1316-1328 (1997).

5. C. M. Mount, D. B. Thiessen, and P. L. Marston, "Scattering observations for tilted transparent fibers: evolution of the Airy caustics with cylinder tilt and the caustic merging transition,” Appl. Opt. 37, 1534-1539 (1998).

6. P. L. Marston, "Descartes glare points in scattering by icicles: color photographs and a tilted dielectric cylinder model of caustic and glare point evolution," Appl. Opt. 37, 1551-1556 (1998).

7. F. J. Blonigen and P. L. Marston, "Backscattering enhancements for tilted solid plastic cylinders in water due to the caustic merging transition: observation and theory," J. Acoust. Soc. Am. 107, 689-698 (2000).

8. J. A. Lock and T. A. McCollum, "Further thoughts on New- ton's zero-order rainbow," Am. J. Phys. 62, 1082-1089 (1994).

9. K. W. Ford and J. A. Wheeler, "Semiclassical description of scattering," Ann. Phys. (N.Y.) 7, 259-286 (1959).

10. M. V. Berry and K. E. Mount, "Semiclassical approximations in wave mechanics," Rep. Prog. Phys. 35, 315-397 (1972); in particular, Sects. 6.1 and 6.2, pp. 335-364.

11. N. Roth, K. Anders, and A. Frohn, "Refractive-index measurements for the correction of particle sizing methods," Appl. Opt. 30, 4960-4965 (1991).

12. J. P. A. J. van Beeck and M. L. Reithmuller, "Nonintrusive measurements of temperature and size of single falling raindrops,” Appl. Opt. 34, 1633-1639 (1995).

13. X. Han, K. F. Ren, Z. Wu, F. Corbin, G. Gouesbet, and G. Grehan, "Characterization of initial disturbances in a liquid jet by rainbow sizing," Appl. Opt. 37, 8498-8503 (1998).

14. Ref. 1, p. 263.

15. W. J. Humphreys, Physics of the Air (Dover, New York, 1964), pp. 476-492.

16. Ref. 1, Appendix 2, pp. 339-342.

17. J. A. Lock, "Scattering of a diagonally incident focused Gaussian beam by an infinitely long homogeneous circular cylinder," J. Opt. Soc. Am. A 14, 640-652 (1997).

18. M. Abramowitz and I. A. Stegun, Handbook of Mathematical Functions (U.S. Government Printing Office, Washington, D.C., 1964), Eq. (9.2.3), p. 447.

19. P. Debye, "Das elektromagnetische Feld um einen Zylinder und die Theorie des Regenbogens," Phys. Z. 9, 775-778 (1908), reprinted and translated into English in P. L. Marston, ed., Geometrical Aspects of Scattering (SPIE Press, Bellingham, Wash., 1994), pp. 198-204.

20. Ref. 18, Eq. (10.4.32), p. 364.

21. T. Pearcey, "The structure of an electromagnetic field in the neighbourhood of a cusp of a caustic," Philos. Mag. 37, 311317 (1946)

22. V. Khare and H. M. Nussenzveig, "Theory of the rainbow," Phys. Rev. Lett. 33, 976-980 (1974).

23. H. M. Nussenzveig, "Complex angular momentum theory of the rainbow and the glory," J. Opt. Soc. Am. 69, 1068-1079 (1979); plates on pp. 1193, 1194.

24. H. M. Nussenzveig, "High-frequency scattering by a transparent sphere. I. Direct reflection and transmission," J. Math. Phys. (N.Y.) 10, 82-124 (1969).

25. H. M. Nussenzveig, "High-frequency scattering by a transparent sphere. II. Theory of the rainbow and the glory," J. Math. Phys. (N.Y.) 10, 125-176 (1969).

26. H. M. Nussenzveig, Diffraction Effects in Semiclassical Scattering (Cambridge U. Press, New York, 1992), pp. 101116.

27. Ref. 18, Eqs. (9.3.15)-(9.3.22), pp. 366-367.

28. H. C. van de Hulst, Light Scattering by Small Particles (Dover, New York, 1981), pp. 208-209.

29. J. D. Walker, "Multiple rainbows from single drops of water and other liquids," Am. J. Phys. 44, 421-433 (1976).

30. R. T. Wang and H. C. van de Hulst, "Rainbows: Mie computations and the Airy approximation," Appl. Opt. 30, 106117 (1991).

31. This result is implicit in P. L. Marston and G. Kaduchak, "Generalized rainbows and unfolded glories of oblate drops: organization for multiple internal reflections and extension of cusps into Alexander's dark band," Appl. Opt. 33, 47024713 (1994). 